\title{
Enfermedad de Behçet
}

Carreño Pérez L. Enfermedad de Behçet. An Med Interna (Madrid) 2001; 18: 401-404.

La triada úlceras orales y genitales recurrentes e iridociclitis descrita por Hulusi Behçet en 1937 es hoy una enfermedad inflamatoria multisistémica que se clasifica entre las vasculitis (1). Su espectro clínico se ha ampliado considerablemente e implica a numerosos órganos y sistemas. Puede aparecer en individuos de todas las edades incluidos recién nacidos (2), con predominio en varones entre la $3^{\mathrm{a}}$ y $4^{\mathrm{a}}$ década de la vida . Su etiología sigue siendo incierta y como sucede habitualmente cuando se trata de enfermedades multisistémicas se han propuesto diversas teorías que pueden resumirse en una combinación de factores genéticos y ambientales y defectos en los mecanismos de inmunoregulación. La prevalencia de HLA B5 entre los pacientes es alta y más específicamente la del alelo B51 que se considera un importante factor de riesgo en áreas en que la incidencia de EB es mayor como sucede en los países asiáticos $(3,4)$. La presencia del HLA B51 también afecta a la severidad de la enfermedad. Es más común entre los pacientes con uveítis posterior o con enfermedad progresiva del sistema nervioso central que en los que tienen una enfermedad leve. Entre los factores ambientales algunas infecciones parecen implicadas en el desarrollo de la enfermedad. Se ha aislado DNA del virus del herpes simple en núcleos de linfocitos de sangre periférica y en el suero se han detectado anticuerpos contra el virus (5). También se ha observado una elevada incidencia de amigdalitis y caries dentales causadas por estreptococos y el estudio histológico de las ulceraciones orales revelaba infiltrados inflamatorios de neutrófilos, linfocitos T y depósitos de componentes de estreptococos (6). Algunas proteínas de choque térmico de los microorganismos presuntamente implicados muestran homologías aminoacídicas con HSP 60 humana, lo que sugiere que los agentes infecciosos a través de mecanismos de similitud molecular con péptidos de tejidos humanos podrían iniciar una respuesta autoinmune. Sin embargo no ha podido demostrarse que alguno de estos agentes infecciosos cause la enfermedad.

La evidencia de anormalidades inmunológicas está bien documentada aunque su relevancia no es suficiente para establecer una teoría autoinmune. En sangre periférica Hagiwara (7) ha encontrado un aumento de células mononucleares Th1 que secretan citoquinas proinflamatorias. Esta alteración se correlaciona con la actividad de la enfermedad. Estas citoquinas podrían estar implicadas en la activación de neutrófilos y en el aumento de interacciones celulares entre los neutrófilos y las células endoteliales incrementando la expresión de moléculas de adhesión (8). La alteración celular característica de la EB es el incremento de migración de los neutrófilos, células predominantes en las lesiones iniciales. El principal hallazgo microscópico de las lesiones activas de la EB es una vasculitis oclusiva mediada por mecanismos inmunes. El infiltrado perivascular revela infiltrados de linfocitos $\mathrm{T}, \mathrm{CD} 4+\mathrm{y} \mathrm{CD} 8+\mathrm{y}$ células HLA-DR (9).

Baixauli y cols. (10) analizan la expresión clínica de la enfermedad y la comparan con otras series de la literatura. Confirman su heterogeneidad y las dificultades del diagnóstico diferencial y comentan las novedades en el tratamiento.

El diagnóstico de la enfermedad sigue basándose en criterios clínicos debido a la ausencia de alteraciones de laboratorio o histológicas patognomónicas. La mayoría de los pacientes pueden clasificarse de acuerdo a los criterios del Grupo Internacional para el Estudio de la EB publicados en 1990 (11). No obstante la existencia de formas incompletas de la enfermedad y de diferencias regionales en la prevalencia de algunas de las manifestaciones clínicas características puede hacer que el diagnóstico se retrase años o no llegue a establecerse. Se precisa la exclusión previa de otras enfermedades que pueden cursar con aftas y manifestaciones reumatológicas como aftosis oral crónica, infecciones herpéticas, síndrome de Sweet, artritis reactivas, enfermedad inflamatoria intestinal.

Las úlceras en mucosas, orales y genitales, el primer criterio diagnóstico, suelen ser la primera manifestación y con frecuencia la única durante años. Aunque se han atribuido ciertas características la mayor parte es indistinguible de otro tipo de úlceras y la histología es inespecífica con evidencia de vasculitis. En los períodos iniciales debe considerarse el diagnóstico diferencial con herpes simple. Otras lesiones cutáneas frecuentes pueden adquirir diversas formas: nodulares semejantes a las del eritema nudoso con rangos histopatológicos de reacción vascular neutrofílica, púrpura palpable, vasculitis necrotizante y pápulas y pústulas como las del acné vulgar o foliculitis. Puesto que estas últimas lesiones son 
inespecíficas, particularmente en adolescentes, algunos autores consideran que no deberían incluirse entre los criterios diagnósticos. Tampoco deben considerarse para el diagnóstico en pacientes que han tomado corticoides (12). El test de patergia incluido entre los criterios de diagnóstico tiene una prevalencia, elevada entre los pacientes del Extremo Oriente, sin embargo es poco frecuente en los del área mediterránea y América y por lo tanto con poca sensibilidad en estas regiones entre las nos incluimos. Su positividad se asocia con un riesgo aumentado de enfermedad vascular (13). No es exclusivo de la EB ya que puede ser positivo en el síndrome de Sweet y en el pioderma gangrenoso. El mecanismo patogénico de esta reacción no es bien conocido y tampoco es exclusivo de la piel. Son varios los tejidos que muestran hiperreactividad a los traumatismos y no es infrecuente la aparición de uveítis tras cirugía ocular o de una sinovitis a continuación de una artrocentesis.

La inflamación ocular, con una prevalencia entre el 47 y $75 \%$ (14), consiste en una uveítis recidivante, en general bilateral, que puede afectar tanto a la cámara anterior como a la posterior o a ambas (15). Suele aparecer después de 2-3 años del comienzo de la enfermedad y en ocasiones es el primer síntoma. En un $20 \%$ de los pacientes puede observarse hipopión que aunque característico no es específico de la EB y puede verse en algunas artropatías asociadas al HLA-B27. La lesión retiniana, la más severa, es una vasculitis con producción de hemorragias y trombosis venosas. Es casi constante el edema macular quístico y la papilitis. Después de cada recidiva queda alguna lesión estructural aunque en los últimos años con el reconocimiento precoz de la enfermedad y los nuevos tratamientos el pronóstico ha mejorado considerablemente. Algunas retinitis virales y la sarcoidosis pueden producir lesiones indistinguibles de las de la EB (16).

Entre el $10 \%$ y $20 \%$ tienen manifestaciones neurológicas. Los cuadros clínicos son meningoencefalitis, signos piramidales y de afección del tronco cerebral y se asocian con peor pronóstico (17). La cefalea aislada no se considera. Las alteraciones del líquido cefalorraquídeo son inespecíficas, con pleocitosis de polimorfonucleares y linfocitos. Con resonancia magnética pueden observarse imágenes focales de hiperseñal en T2, en el tronco cerebral, ganglios basales y sustancia blanca. Estas imágenes no son específicas de la EB y aparecen en otras enfermedades que cursan con vasculitis. Las alteraciones psiquiátricas son frecuentes y en los períodos terminales la demencia comienza a la hacerse evidente en estos pacientes.

En el aparato digestivo la ulceración del tracto gastrointestinal es la lesión característica y la localización más frecuente la mucosa del íleon terminal y ciego y, en menor grado, de otros territorios del colon. También puede afectarse el esófago. El diagnóstico diferencial con otras enfermedades intestinales, en particular con la colitis ulcerosa, no es fácil. Los cambios histológicos son similares y las manifestaciones extraarticulares, como ulceras orales, eritema nudoso, uveítis $\mathrm{y}$ artritis, indistinguibles por lo que es preciso excluir una enfermedad inflamatoria intestinal antes de establecer el diagnóstico de EB.

La mitad de los pacientes con EB tiene artritis, oligo o monoarticular, no erosiva que afecta a las grandes articulaciones de los miembros inferiores. La artritis no se limita a las articulaciones periféricas. Dilsen (18) encuentra sacroileítis en el $34 \%$ de 331 pacientes turcos, en general sin dolor lum- bar inflamatorio y Baixauli utilizando tomografía computarizada en el $41 \%$ de sus pacientes también con poca sintomatología. Curiosamente Graña (19) solo la encuentra en 3 de 109 pacientes gallegos. Es debido a esta elevada prevalencia de sacroileítis que la EB fuera clasificada inicialmente entre las espondiloartropatías seronegativas. Quizá los pacientes con sacroileítis B27 (+) deberían ser incluidos en el espectro de artritis reactiva o en el de enfermedad inflamatoria intestinal en lugar de EB.

La afección vascular tiene una clara predisposición masculina y afecta tanto a arterias como a venas de todos los tamaños y territorios. A pesar de su frecuencia e importancia sigue discutiéndose si la participación de los grandes vasos, que se produce entre el 15 y el $35 \%$ de los pacientes con EB, debería incluirse entre los criterios de diagnóstico de la enfermedad (20). Los grandes vasos puede afectarse precozmente incluso antes de que aparezcan las úlceras orales, sin otras manifestaciones clínicas, por lo que el reconocimiento de la enfermedad en estos casos es muy problemático. Koç (13) en su estudio sobre 137 pacientes y revisión de otros 728 de la literatura distingue tres formas de enfermedad vascular: oclusiones venosas (88\%), aneurismas arteriales y/o oclusiones arteriales $(12 \%)$. La lesión venosa más frecuente era la tromboflebitis subcutánea. El desarrollo de trombosis superficial se asocia con riesgo elevado de presentarse en otras localizaciones y de lesiones arteriales. De éstas $2 / 3$ son aneurismas que pueden observarse en todo el árbol arterial incluidas las coronarias y $1 / 3$ son oclusiones. La arteria que se afecta con mayor frecuencia es la aorta seguida de la pulmonar, femoral, poplítea, subclavias y carótidas. La combinación de tromboflebitis con aneurismas arteriales pulmonares, síndrome de Hughes Stovin, se ha sugerido como manifestación vascular única (1). El retraso en el diagnóstico y en el inicio del tratamiento de esta complicación puede ser responsable de la rotura del aneurisma con hemoptisis masiva con una elevada mortalidad. La base patológica es una vasculitis de los vasa vasorum.

La causa de la tendencia a la trombosis en la EB no es bien conocida y tampoco la importancia de las alteraciones de la coagulación observadas. La elevación de los niveles de antitrombina III que muestran algunos pacientes puede deberse a mecanismos compensatorios contra el incremento de la actividad procoagulante en pacientes con tromboembolismo (21).

La participación de otros órganos es menos frecuente. Se han descrito casos de pericaditis, endocarditis, infartos de miocardio y vasculitis coronaria y aneurismas ventriculares. Las alteraciones pulmonares, aneurismas arteriales, trombosis venosa y arterial, infartos pulmonares, también están relacionadas con vasculitis. Rara vez se ve derrame pleural. La TAC puede ayudar al diagnóstico de los aneurismas. Como en otros territorios los estudios histológicos de las lesiones arteriales muestran arteritis de los vasa vasorum. También se han descrito alteraciones renales que van desde glomerulonefritis difusa y focal sin alteración de la función renal a proteinuria y hematuria microscópica.

La EB también puede aparecer en la infancia, en general con formas incompletas. Las manifestaciones más comunes son las úlceras orales y genitales, lesiones cutáneas, artritis y uveítis. Algunos recién nacidos de mujeres con EB presentan úlceras orales y genitales, pústulas y úlceras necróticas cutáneas de distribución periungueal (2). Estas lesiones son autolimitadas y desaparecen en seis semanas. El curso de la EB 
durante el embarazo es variable. Puede producirse activación, remisión o cambios clínicos en cada caso. Gül (22) en un estudio con 16 mujeres con EB durante la gestación y posterior parto, observó que las manifestaciones clínicas de actividad durante el embarazo consisten en lesiones mucocutáneas y artritis. Inflamaciones oculares y tromboflebitis se produjeron en pocas ocasiones. Todos los embarazos llegaron a término y los niños nacieron sanos. Las reactivaciones post-parto fueron escasas.

El tratamiento, con frecuencia poco satisfactorio, es sintomático y depende de las manifestaciones clínicas. A la hora de planificarlo hay que tener en cuenta que la enfermedad cursa con remisiones y exacerbaciones impredecibles, que el comienzo a edad temprana y el sexo masculino son factores de mal pronóstico y que por tanto requieren una intervención precoz y enérgica que evite el daño estructural irreversible y que algunos pacientes con enfermedad leve no precisan tratamiento. La participación de órganos vitales marca la toma de decisiones. Las prioridades son las complicaciones gastrointestinales, las del sistema nervioso central y las de los grandes vasos que con frecuencia requieren dosis elevadas de corticoides e inmunosupresores y en ocasiones intervenciones quirúrgicas.

La lesiones mucocutáneas responden en general al tratamiento con corticoides tópicos o intralesionales. La colchicina, usada en el tratamiento de las lesiones mucocutáneas de la EB desde el año 1975, resulta útil debido a su acción inhibitoria sobre la función de los neutrófilos. La talidomida, que a dosis de $100 \mathrm{mg} /$ día muestra la misma efectividad que 300 $\mathrm{mg} /$ día, ha demostrado ser muy eficaz en la resolución de las lesiones mucocutáneas refractarias a otros tratamientos. $\mathrm{Su}$ mecanismo de acción es a través de la inhibición del TNF- $\alpha$ y de la angiogénesis (23). Su mayor inconveniente es la teratogenicidad y la neuropatía periférica que produce. La dapsona oral a dosis entre 50 y $100 \mathrm{mg}$ /día también ha resultado ser útil en pacientes con manifestaciones mucocutáneas persistentes aunque su uso en la EB es muy restringido (24). En casos de enfermedad mucocutánea grave están indicados los corticoides sistémicos. El tratamiento de las lesiones oculares depende de su severidad. Comienzo temprano y sexo masculino son factores de riesgo de complicaciones oculares severas. Los agentes citotóxicos o inmunosupresores como azatioprina, clorambucilo, ciclofosfamida pueden prevenir la aparición de nuevos episodios en el $50 \%$ de los pacientes (25). La ciclofosfamida es la más eficaz en la vasculitis retiniana. En pacientes refractarios a otros tratamientos la ciclosporina sola o en combinación con azatioprina puede ser eficaz. Este tratamiento está contraindicado en pacientes con síntomas neurológicos y con lesiones neurálgicas subclínicas detectadas por resonancia magnética porque puede ser neurotóxica en pacientes con EB. Recientes estudios han mostrado esperanzadores resultados con interferón- $\alpha$ aunque no se conoce la dosis adecuada y se produce una reactivación al suspender el tratamiento (26). El interferón- $\alpha$ también produjo una marcada reducción de las lesiones mucocutáneas y de la vasculitis. De la misma forma, los anticuerpos anti-TNF- $\alpha$ han demostrado ser muy eficaces en la supresión de la uveítis posterior refractaria, como acaban de comunicar Bañares y cols. (27).

El tratamiento en los pacientes con oclusiones venosas continua siendo controvertido. Va desde el uso de antiagregantes plaquetarios hasta anticoagulación oral o parenteral (20). Los pacientes con vasculitis y aneurismas pueden beneficiarse de la terapia con ciclofosfamida oral o en pulsos mensuales, sola o en combinación con esteroides sistémicos (8). Las lesiones gastrointestinales requieren sulfasalacina y esteroides. La cirugía se considerará en los pacientes con perforación intestinal y sangrado persistente. Para prevenir complicaciones postquirúrgicas por la reacción inflamatoria local característica en la $\mathrm{EB}$, los pacientes deben recibir esteroides durante varios días. El tratamiento de las fases agudas de la enfermedad neurológica se basa en altas dosis de esteroides, orales o en pulsos intravenosos, seguidos de dosis medias orales durante varios meses. Pueden asociarse ciclofosfamida o metotrexato. La enfermedad progresiva crónica del sistema nervioso central es en general resistente a las terapias disponibles hoy.

En conclusión en los últimos años se han producido importantes avances en el tratamiento de la EB particularmente en el tratamiento de la uveítis, de las lesiones mucocutáneas y de las vasculares. No obstante el pronóstico de los pacientes sigue siendo impreciso y el $25 \%$ de los que tienen lesiones oculares pueden perder la visión. Las causas de muerte siguen siendo la ruptura de aneurismas, la enfermedad severa del SNC y la perforación intestinal por lo que es imprescindible identificar precozmente estas complicaciones para aplicar medidas terapéuticas adecuadas. Sorprendentemente algunos pacientes con trombosis masiva de la cava pueden sobrevivir durante años al desarrollar una extensa red colateral de venas superficiales y varices. Solo el conocimiento de los mecanismos patogénicos permitirá una actuación terapéutica más eficaz en el futuro.

\section{CARREÑO PÉREZ}

Servicio de Reumatología. Hospital General Universitario Gregorio Marañón. Madrid

\section{Bibliografía}

1. Lie JT. Vascular involvement in Behçet Disease: Arterial and venous and vessels of all sizes. J Rheumatol 1992; 19: 341-342

2. Lewis MA, Priestly BL. Transient neonatal Behçet disease. Arch Dis Child. 1986; 61: 805-806.

3. Yacici H, Yurdakul S, Hamuryundan V. Behçet syndrome. In Oxford textbook of Rheumatology. 2 ed. Ed. Oxford: Oxford University Press, 1998; 1394-1402.

4. Kaklamani VG, Vaiopoulos G, Kaklamanis PG. Becçet's disease. Semin Arthritis Rheum 1998; 27: 197-217.

5. Lee S, Bang D, Cho YH. Polymerase chain reaction reveals herpes simplex virus DNA in saliva of patients with Behçet's disease. Arch Dermatol Resch 1996; 288: 179-183

6. Mizushima Y, Matsuda T, Hoshi K, et al. Induction of Behçet's disease symptoms following dental treatment and streptococcal antigen skin test. J Rheumatol 1998; 15: 1029-1030.

7. Hagiwara E, Okubo T, Misumi M, et al. Altered frecuency of cytokine secreting cells in patients with Behçet disease. Arthritis Rheum 2000; 43 (suppl): s 363. 
8. Ehrlich GE. Vasculitis in Behçet disease. Int Rev Inmunol 1997; 14: 8188

9. Celenligil H, Kansu E, Ruancan S, et al. Characterization of peripheral blood lymphocytes and immunohistological analysis of oral ulcers in Behçet's disease. In: O’Duffy JD, Kökmen E eds. Behçet's Disease. New York: Marcel Dekker 1991: 487-496.

10. Baixauli A, Calvo J, Tamarit JJ, Campos C, García S, Herrera A. Enfermedad de Behçet: estudio retrospectivo. An Med Interna (Madrid) 2001; 18: 405-410.

11. International Study Group for Behçet's Disease. Criteria for diagnosis of Behçet's disease. Lancet 1990; 335: 1078-1080.

12. Ghate JV, Jorizzo JL. Behçet's disease and complex aphthosis. J Am Acad Dermatol 1999; 40:1-18.

13. Koç Y, Güllü I, Akpek G, et al. Vascular Involvement in Behçet Disease. J Rheumatol 1992; 19: 402-410.

14. Sakane T, Takeno M, Suzuki N, et al. Behçet's Disease. N Engl J Med 1999; 341: 1284-1291

15. Candelas Rodríguez G, Macarrón Pérez P, Jover Jover JA. Manifestaciones oculares de la enfermedad de Behçet y otras vasculitis sistémicas. Rev Esp Reumatol 1997; 24: 185-189.

16. Nussemblatt RB. Uveitis in Behçet's disease. Int Rev Immunol 1997; 14: 67-79.

17. Serdaroglu P. Behçet's disease and the Nervous System. J Neurol 1998; 245: 197-205.

18. Dilsen N, Koniçe M, Aral O. Why Behçet's disease should be accepted as seronegative arthritis. In: Lehner T, Barnes CG eds. Recent advances in Behçet ${ }_{j}$ s disease. International Congress and Symposium series. $\mathrm{N}^{\mathrm{o}}$
103. London/New York: Royal Society of Medicine Services; $1986 \mathrm{p}$ 281-284.

19. Graña Gil J, Galdo Fernández F. Enfermedad de Behçet. Otras vasculitis. Procesos que simulan vasculitis sistémica. En Manual SER de la Enfermedades Reumáticas. Madrid: Ed. Médica Panamericana S.A. $2000 ; 377-387$.

20. Schirmer M, Calamia KT, O’Duffy JD. Is there a place for large vessel disease in the diagnostic criteria of Behçet's disease? J Rheumatol 1999; 26: 2511-2512.

21. Sengul N, Demirer S, Yerdel MA, et al. Comparison of coagulation parameters for healthy subjects and Behçet disease patient with and wit hout vascular involvement. World J Surg 2000; 24: 1584-1588.

22. Gül Ü. Pregnancy and Behçet Disease. Arch Dermatol 2000; 136: 1063 1064

23. Calabrese L, Resztak K. Thalidomida revisited: Pharmacology and clinical applications. Expert Opin Invest Druggs. 1998; 7 (12): 2043-2060.

24. Kaklamani VG, Kaklamanis PG. Treatment of Behçet's Disease-An Update. Semin Arthritis Rheum 2001; 30: 299-312.

25. Robertson LP, Hickling P. Treatment of recalcitrant urogenital ulceration of Behçet's syndrome with infliximab. Rheumatology 2001; 40(4): 473-474.

26. Zouboulis CC, Orfanos CE. Treatment of Adamantiades-Behçet's disease with systemic interferon alpha. Arch Dermatol 1998; 134: 10101016.

27. Bañares A, Pato E, Abasolo L, et al. Treatment of refractory posterior uveítis with anti-TNF alfa (Infliximab). Ann Rheum Dis 2001; 60 (suppl 1):145. 